\title{
Direct comparison of Xpert Xpress, FilmArray Respiratory Panel, Lumipulse antigen test, and RT-qPCR in 165 nasopharyngeal swabs
}

\author{
Yosuke Hirotsu ${ }^{1 *}$ (D, Makoto Maejima², Masahiro Shibusawa², Yume Natori ${ }^{2}$, Yuki Nagakubo 2,3, \\ Kazuhiro Hosaka² ${ }^{2}$ Hitomi Sueki ${ }^{2}$, Kenji Amemiya ${ }^{3}$, Miyoko Hayakawa ${ }^{4}$, Hitoshi Mochizuki ${ }^{1,4,5}$, Toshiharu Tsutsui ${ }^{6}$, \\ Yumiko Kakizaki ${ }^{6}$, Yoshihiro Miyashita ${ }^{6}$ and Masao Omata ${ }^{5,7}$
}

\begin{abstract}
Background: The nucleic acid amplification test (NAAT) and antigen test are approved diagnostic tests for COVID-19. In this study, we aimed to investigate the assay performance of two NAATs (Xpert Xpress SARS-CoV-2 and FilmArray Respiratory Panel) and a quantitative antigen test (Lumipulse).

Methods: One hundred and sixty-five nasopharyngeal swabs were subjected to Xpert, FilmArray, Lumipulse, and RT-qPCR assays.

Results: Of 165 samples, RT-qPCR showed 100 positives and 65 negatives. The Xpert had an overall agreement of 99.4\% (95\% confidence interval [Cl]: 96.7-99.4\%), sensitivity of 99\% (95\% Cl: 96.8-99\%), and specificity of 100\% (95\% Cl: 96.6-100\%). FilmArray had an overall agreement of 98.8\% (95\% Cl: 95.9-98.8\%), sensitivity of 98\% (95\% Cl: 95.698\%), and specificity of 100\% (95\% Cl: 96.3-100\%). Lumipulse had an overall agreement of 95.5\% (95\% Cl: 91.8-95.5\%), sensitivity of $92.3 \%$ (95\% Cl: 89.2-92.3\%), and specificity of 100\% (95\% Cl: 95.5-100\%). The k coefficient showed excellent agreement between each test and RT-qPCR. There was a high correlation between Xpert Ct values, RT-qPCR Ct values, viral loads and antigen level.

Conclusions: Xpert Xpress and FilmArray Respiratory Panel exhibited an equivalent performance. The Lumipulse antigen test was slightly less sensitive than the NAATs, but showed high assay performance except for samples with low viral load. The Xpert Xpress, FilmArray Respiratory Panel and Lumipulse antigen tests offer rapid sample-to-answer data, allowing random access detection on automated devices.
\end{abstract}

Keywords: SARS-CoV-2, COVID-19, Xpert Xpress, FilmArray, Lumipulse

\section{Background}

The emergence of novel Variants of Concern (VOCs) is threatening human life [1-5]. In particular, the spread of the Alpha, Beta, Gamma, Delta and Omicron variant has led to an infection outbreak in several countries [6-8].

\footnotetext{
*Correspondence: hirotsu-bdyu@ych.pref.yamanashi.jp

1 Genome Analysis Center, Yamanashi Central Hospital, 1-1-1 Fujimi, Kofu, Yamanashi, Japan

Full list of author information is available at the end of the article
}

Therefore, diagnostic tests for coronavirus disease 2019 (COVID-19) continue to be in high demand.

Reverse transcription-polymerase chain reaction (RTPCR) is the standard method for detecting severe acute respiratory syndrome coronavirus 2 (SARS-CoV-2) [9]. The steps of RT-PCR are as follows: extract genomic RNA from the virus, reverse transcribe into complementary DNA, amplify the region of interest, and detect fluorescent signals. This method amplifies and detects viral-derived nucleic acids, making it possible to 
sensitively evaluate samples with low viral loads. However, when performed manually, it requires skilled techniques and hands-on time, taking approximately $3-4 \mathrm{~h}$ to obtain the results.

Automated devices for testing SARS-CoV-2 have been developed. Random access detection with automated devices enables the rapid return of results to clinicians and patients. In addition, once the sample is loaded into the dedicated reagent, the process from sample to answer is seamless. These tests are straightforward to perform and do not always require skilled personnel. In addition, random access detection can be performed for each sample, making it useful for emergency testing.

We previously showed that the FilmArray Respiratory Panel (v2.1) and Lumipulse antigen test had high accuracy for detecting SARS-CoV-2 in nasopharyngeal swab samples [10-12]. Next, we planned to strengthen our testing system for SARS-CoV-2 by installing Xpert Xpress SARS-CoV-2. Previous studies reported the performance of Xpert compared with that of the Cobas SARS-CoV-2 assay or in-house real-time RT-PCR assays using clinical samples [13-17]. However, there have been no reports of a head-to-head comparison between the Xpert and FilmArray or Lumipulse. In this study, we aimed to evaluate the assay performance of the Xpert Xpress SARS-CoV-2 and FilmArray Respiratory Panel as automated RT-PCR assays and tested the Lumipulse as an automated antigen quantification test. The same samples were also tested for quantitative RT-PCR (RT-qPCR) as the reference, and the results were compared.

\section{Methods}

\section{Patients and samples}

A total of 165 nasopharyngeal swabs were collected and stored in viral transport medium (VTM) (Copan, Murrieta, CA, USA). The patients comprised 78 women and 87 men, with a mean age of 49.2 years (range 1-94). The Institutional Review Board at Yamanashi Central Hospital approved this study and the use of an opt-out consent method (G-2019-1). The requirement for written informed consent was waived because this was an observational study. Patient participation in the study was optional.

\section{Viral nucleic acid extraction}

We extracted total nucleic acid from $200 \mu \mathrm{L}$ of VTM using a MagMax Viral/Pathogen Nucleic Acid Isolation Kit (Thermo Fisher Scientific, Waltham, MA, USA) on a KingFisher Duo Prime (Thermo Fisher Scientific) as previously described $[18,19]$. These extracted nucleic acids were analyzed by quantitative reverse transcription PCR (RT-qPCR). The residual VTM was used for testing with the Xpert Xpress SARS-CoV-2, FilmArray Respiratory
Panel, and Lumipulse SARS-CoV-2 antigen test as described below.

\section{RT-qPCR}

To detect SARS-CoV-2, we performed one-step RTqPCR in accordance with the protocol developed by the National Institute of Infectious Diseases in Japan [20]. This method amplifies the nucleocapsid $(N)$ gene of SARS-CoV-2 (NC_045512.2) [9]. We made reaction mixture composed of $5 \mu \mathrm{L}$ of $4 \times$ TaqMan Fast Virus 1-Step Master Mix (Thermo Fisher Scientific), $1.0 \mu \mathrm{L}$ of $10 \mu \mathrm{M}$ forward primer, $1.4 \mu \mathrm{L}$ of $10 \mu \mathrm{M}$ reverse primer, $0.8 \mu \mathrm{L}$ of $5 \mu \mathrm{M}$ probe, $6.8 \mu \mathrm{L}$ of nuclease-free water, and $5 \mu \mathrm{L}$ of the nucleic acid sample in a $20-\mu \mathrm{L}$ total volume as previously described [9].

The StepOnePlus Real-Time PCR System (Thermo Fisher Scientific) was used for RT-qPCR assays as following condition: $50{ }^{\circ} \mathrm{C}$ for $5 \mathrm{~min}, 95^{\circ} \mathrm{C}$ for $20 \mathrm{~s}$, and 45 cycles of $95{ }^{\circ} \mathrm{C}$ for $3 \mathrm{~s}$ and $60{ }^{\circ} \mathrm{C}$ for $30 \mathrm{~s}$. The threshold cycle was examined then the threshold was set at 0.2 . We judged the results according to the protocol (version 2.9.1) [20].

\section{Xpert Xpress SARS-CoV-2}

The Xpert Xpress SARS-CoV-2 (Cepheid, Sunnyvale, CA) is an automated real-time RT-PCR test carried out in single-use disposable cartridges. For this assay, $300 \mu \mathrm{L}$ of VTM was loaded into the sample chamber of the cartridge using a sterile pipette. The cartridges were set in a GeneXpert System (Cepheid) and analyzed. This assay targets the envelope $(E)$ gene and $N$ gene (named N2) and detects SARS-CoV-2 according to the cycle threshold cut-off values.

\section{FilmArray Respiratory Panel}

We performed multiplex real-time RT-PCR assay using the FilmArray Respiratory Panel version 2.1 (bioMérieux, Marcy-l'Etoile, France) [21]. This assay detects 21 pathogens (i.e., SARS-CoV-2 and 17 other viruses and three bacteria) related to respiratory diseases. The kit buffer and $300 \mu \mathrm{L}$ of VTM were injected into the FilmArray pouch and the reaction proceeded on the FilmArray Torch system (bioMérieux).

\section{Lumipulse SARS-CoV-2 antigen test}

The SARS-CoV-2 antigen levels were determined quantitatively with the Lumipulse SARS-CoV-2 antigen test (Fujirebio, Inc., Tokyo, Japan) as previously described [12]. Briefly, $700 \mu \mathrm{L}$ of the VTM were vortexed and centrifuged at $2000 \times g$ for $5 \mathrm{~min}$. Aliquots $(100 \mu \mathrm{L})$ of the supernatant were tested on the LUMIPULSE G600II system (Fujirebio). For samples with an antigen level $>5000 \mathrm{pg} / \mathrm{mL}$, the samples were diluted with the kit 


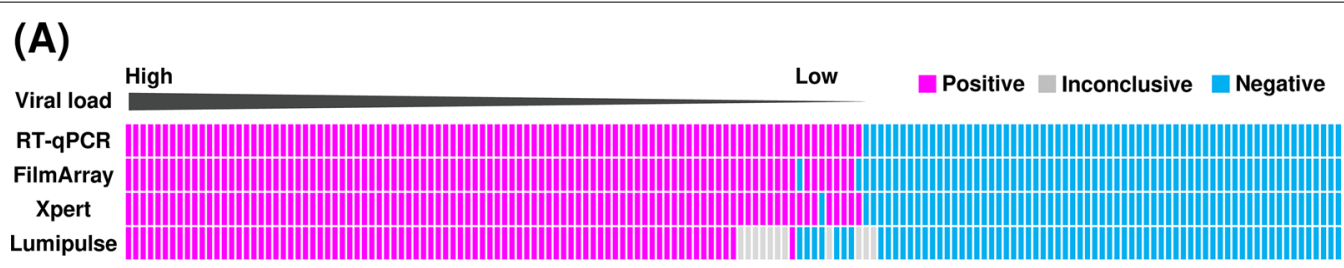

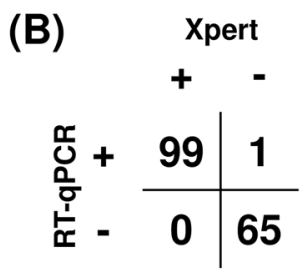

Overall agreement: $99.4 \%$

Sensitivity: $99 \%$

Specificity: $100 \%$

coefficient $=0.987$

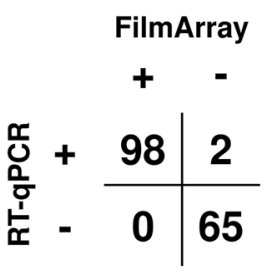

Overall agreement: $\mathbf{9 8 . 8 \%}$

Sensitivity: $98 \%$

Specificity: 100\%

K coefficient $=0.975$

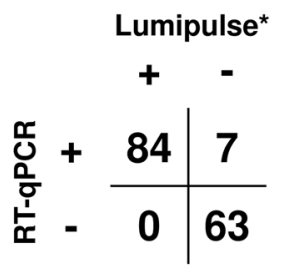

Overall agreement: $\mathbf{9 5 . 5} \%$

Sensitivity: $92.3 \%$

Specificity: $100 \%$

coefficient $=0.909$

Fig. 1 Comparison of RT-qPCR, Xpert, FilmArray, and Lumipulse test results. A Analysis of 165 nasopharyngeal swabs using RT-qPCR, with data shown from the highest viral load (left) to the lowest (right). Pink in the box indicates positive, gray indicates inconclusive, and light blue indicates negative. B Comparison of Xpert, FilmArray, and Lumipulse with RT-qPCR results as a reference. ${ }^{*}$ The Lumipulse data exclude 11 inconclusive samples. + positive; - negative

diluent and tested again, and the antigen level was calculated based on the dilution factor. We judged the results according to the manufacturer's instruction.

\section{Statistical analysis}

Sensitivity and specificity values were calculated using the RT-qPCR results as the reference and with the exclusion of inconclusive samples determined by Lumipulse antigen testing. Cohen's kappa (к) coefficient of results between the two tests with $95 \%$ confidence intervals (CI) was calculated. Cohen's $\mathrm{K}$ values $>0.81$ were interpreted to indicate near-perfect agreement [22]. Statistical analysis was performed using R Version 3.1.2 (http://www.rproject.org/).

\section{Results}

\section{Test accuracy}

A total of 165 nasopharyngeal swab samples were analyzed to investigate the accuracy of test results. Of the 165 samples, 100 were RT-qPCR positive, and 65 were RT-qPCR negative (Fig. 1A). Using these samples, Xpert, FilmArray, and Lumipulse tests were conducted.

When the results of RT-qPCR were used as a reference, the overall agreement rate of Xpert was $99.4 \%$ (95\% CI: 96.7-99.4\%) with a sensitivity of $99 \%$ (95\% CI: 96.8-99\%) and specificity of $100 \%$ (95\% CI: $96.6-100 \%$ ) (Fig. 1B). The overall agreement rate of FilmArray was 98.8\% (95\% CI: 95.9-98.8\%) with a sensitivity of $98 \%$ (95\% CI: 95.6-98\%) and specificity of 100\% (95\% CI: 96.3-100\%) (Fig. 1B).
When inconclusive results $(n=11)$ were excluded for the Lumipulse test (Fig. 1A), the overall agreement was 95.5\% (95\% CI: 91.8-95.5\%) with a sensitivity of 92.3\% (95\% CI: $89.2-92.3 \%$ ) and specificity of $100 \%$ (95\% CI: $95.5-100 \%)$ (Fig. 1B). The $\mathrm{\kappa}$ coefficient indicated excellent agreement between each test and RT-qPCR (Xpert vs RT-qPCR, $\kappa=0.987$, 95\% CI: 0.932-0.987; FilmArray vs RT-qPCR, $\kappa=0.975,95 \%$ CI: 0.914-0.975; Lumipulse vs RT-qPCR, $\kappa=0.909$, 95\% CI: 0.836-0.909). Compared to Lumipulse, Xpert and FilmArray were equivalent in accuracy to RT-qPCR.

\section{Detectable range and discordant results}

To examine the detectable range of viral loads, the results of three PCR tests (RT-qPCR, Xpert, and FilmArray) were compared. There were 97 samples with positive results determined by all three tests (Fig. 2 and Table 1). Among these 97 positive samples with three tests, the mean viral load was $5.0 \log _{10}$ copies/mL (0.2-8.0 $\log _{10}$ copies $/ \mathrm{mL}$ ), the mean RT-qPCR Ct value was 21.5 (range 12-40), the mean Xpert Ct value (N2) was 23.7 (range 14.2-42.6), and the mean Xpert Ct value (E) was 21.7 (range 12.6-42) (Table 1).

Of 100 RT-qPCR positive samples, there were 91 samples with RT-qPCR Ct values $<35$ and 9 samples with $\mathrm{Ct}$ values $\geq 35$ (Fig. 2). Among 91 samples with $\mathrm{Ct}<35$, all samples were detectable with both Xpert and FilmArray (Fig. 2). Among nine samples with $\mathrm{Ct} \geq 35$, six samples were detected by both Xpert and FilmArray, one sample 


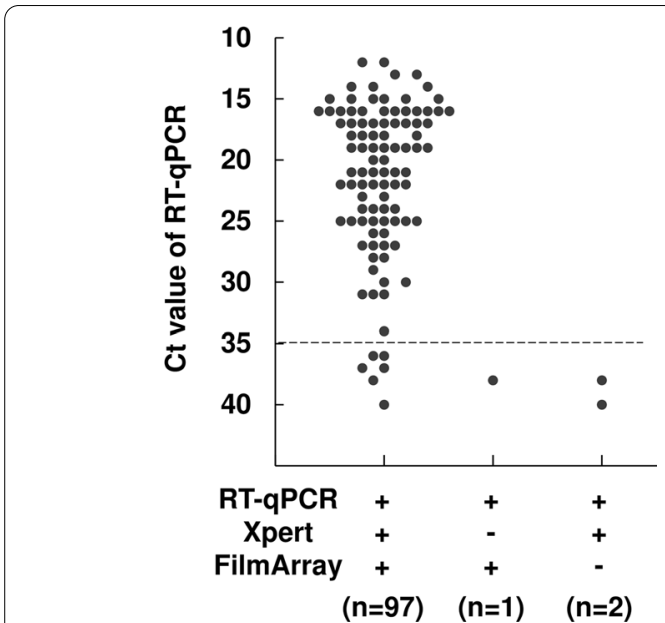

Fig. 2 Relationship between $C t$ values and RT-qPCR results. Ninety-seven samples were positive for all tests (RT-qPCR, Xpert, and FilmArray), one sample was negative for Xpert only, and two samples were negative for FilmArray only. The dotted line indicates a Ct value of $35 .+$ positive; - negative

was not detected by Xpert (the viral load was $0.8 \log _{10}$ copies/mL and the RT-qPCR Ct value was 38 ), and two samples were not detected by FilmArray (viral loads of
1.4 and $0 \log _{10}$ copies/mL and RT-qPCR Ct values of 38 and 40, respectively) (Fig. 2 and Table 2). Therefore, all discordant samples had very low viral loads.

\section{Correlation between antigen level, $\mathrm{Ct}$ values and viral loads}

The FilmArray is a qualitative test, whereas Xpert targets two different genes, including $N$ and $E$, and yields $\mathrm{Ct}$ values. Thus, we examined the correlation between the Xpert Ct values, RT-qPCR Ct values, and viral loads. The results showed that the Xpert $\mathrm{Ct}$ values were highly correlated with the RT-qPCR Ct values for both $\mathrm{N} 2$ and $\mathrm{E}$ (Fig. 3A and B; $\mathrm{R}^{2}=0.977$, Xpert Ct (N2) vs RT-qPCR Ct; $\mathrm{R}^{2}=0.935$, Xpert Ct (E) vs RT-qPCR Ct). In addition, the viral load measured by RT-qPCR and the Xpert Ct value also showed a high correlation (Fig. $3 C$ and $D ; R^{2}=0.956$, Xpert Ct (N2) vs viral load; $R^{2}=0.912$, Xpert Ct (E) vs viral load). Because RT-qPCR amplifies the $N$ gene, the data of Xpert N2 were shown to have a higher correlation than $\mathrm{E}$. These results indicate that the Xpert Ct value accurately reflected the viral load in nasopharyngeal swabs.

We also examined the correlation between the antigen levels, viral loads, RT-qPCR Ct values, and Xpert $\mathrm{Ct}$ values. As we previously reported [12], there

Table 1 Results of RT-qPCR, Xpert, and FilmArray

\begin{tabular}{|c|c|c|c|c|c|c|c|}
\hline RT-qPCR & Xpert & FilmArray & $\begin{array}{l}\text { Number } \\
\text { of samples } \\
(n=165)\end{array}$ & $\begin{array}{l}\text { Mean viral loads of RT-qPCR } \\
{\left[\log _{10} \text { copies } / \mathrm{mL}\right] \text { (range) }}\end{array}$ & $\begin{array}{l}\text { Mean RT-qPCR Ct } \\
\text { value (range) }\end{array}$ & $\begin{array}{l}\text { Mean Xpert Ct } \\
\text { value (N2) (range) }\end{array}$ & $\begin{array}{l}\text { Mean Xpert } \\
\text { Ct value (E) } \\
\text { (range) }\end{array}$ \\
\hline Pos & Pos & Pos & 97 & $5.0(0.2-8.0)$ & $21.5(12-40)$ & $23.7(14.2-42.6)$ & $21.7(12.6-42)$ \\
\hline Pos & Pos & Neg & 2 & $0.7(0-1.4)$ & $39(38-40)$ & $40.4(39.1-41.6)$ & $44.1^{*}$ \\
\hline Pos & Neg & Pos & 1 & 0.8 & 38 & NA & NA \\
\hline Pos & Neg & Neg & 0 & NA & NA & NA & NA \\
\hline Neg & Pos & Pos & 0 & NA & NA & NA & NA \\
\hline Neg & Pos & Neg & 0 & NA & NA & NA & NA \\
\hline Neg & Neg & Pos & 0 & NA & NA & NA & NA \\
\hline Neg & Neg & Neg & 65 & NA & NA & NA & NA \\
\hline
\end{tabular}

Pos positive, Neg negative, $R T$ - $q P C R$ quantitative reverse transcriptase polymerase chain reaction, $C t$ threshold cycle, $N$ nucleocapside, $E$ envelope, $N A$ not applicable *One of the two samples did not exceed the threshold, and the $\mathrm{Ct}$ value could not be determined

Table 2 Samples with discrepant test results

\begin{tabular}{llllll}
\hline No & RT-qPCR & Xpert & FilmArray & $\begin{array}{l}\text { Viral loads of RT-qPCR (log } \\
\text { copies/mL) }\end{array}$ & $\begin{array}{l}\text { Ct value of RT-qPCR } \\
\text { of value } \\
\text { (N2/E) }\end{array}$ \\
\hline 1 & Pos & Neg & Pos & 1.4 & 38 \\
2 & Pos & Pos & Neg & 0.8 & 38 \\
3 & Pos & Pos & Neg & 0 & $39.1 / 44.1$ \\
\hline
\end{tabular}

Pos positive, Neg negative, $R T$ - $q P C R$ quantitative reverse transcriptase polymerase chain reaction, $C t$ threshold cycle, $N$ nucleocapside, $E$ envelope

NA not applicable 
(A)

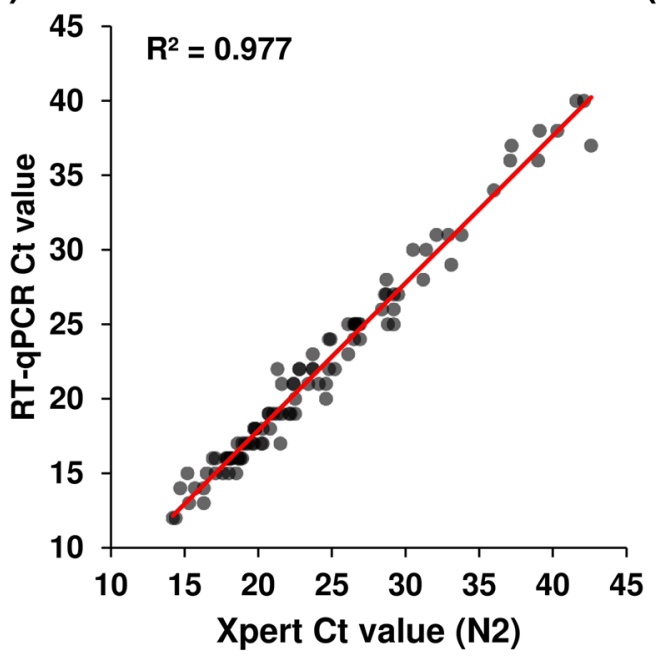

(C)

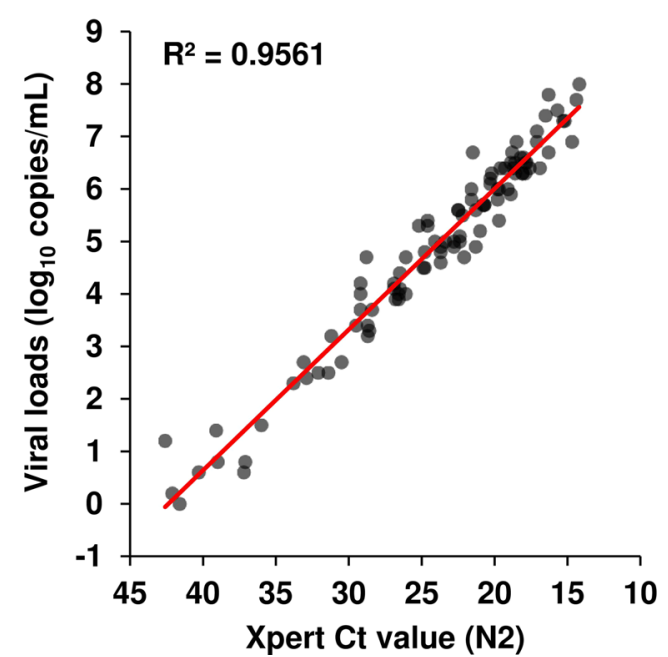

(B)

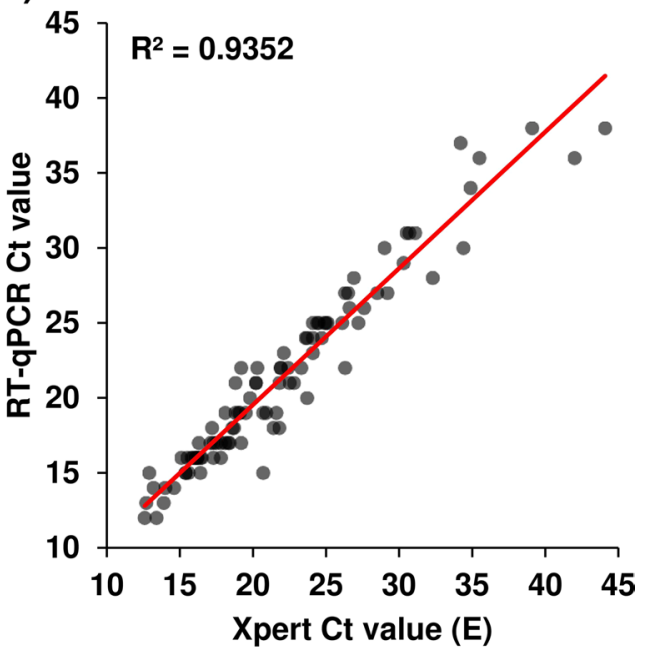

(D)

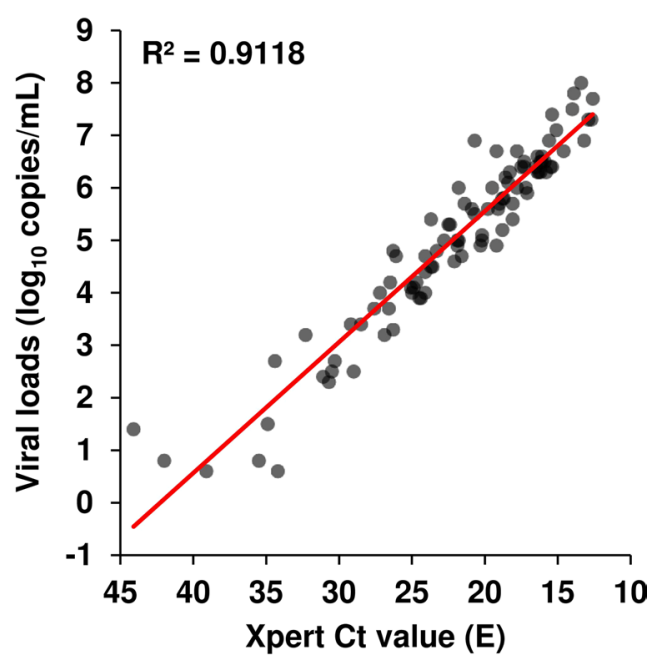

Fig. 3 Correlation between the Ct value of RT-qPCR, viral load, and Ct value of Xpert. A, B Correlation between the Ct value of RT-qPCR and the Ct value of Xpert. C, D Correlation between the viral load determined by RT-qPCR and the Ct value of Xpert. Xpert was plotted for the Ct values of N2 ( $\mathbf{A}$ and $\mathbf{C}$ ) and $\mathbf{E}$ (B and $\mathbf{C}$ ). The decision coefficient $\left(\mathrm{R}^{2}\right)$ is shown in the scatter plot

was a correlation between antigen levels and viral loads $\left(\mathrm{R}^{2}=0.893\right.$, Fig. $\left.4 \mathrm{~A}\right)$ or $\mathrm{RT}-\mathrm{qPCR} \mathrm{Ct}$ values $\left(R^{2}=0.902\right.$, Fig. 4B). Similarly, there was a correlation between the antigen level and the Xpert $\mathrm{Ct}$ values in N2 $\left(R^{2}=0.888\right.$, Fig. $\left.4 C\right)$ and $E$ regions $\left(R^{2}=0.877\right.$, Fig. $\left.4 D\right)$.

\section{Discussion}

We evaluated the performance of the newly installed Xpert system in our hospital. The results showed the test performance of Xpert was almost equivalent to that of FilmArray or RT-qPCR. Meanwhile, the Lumipulse antigen test showed a slightly lower detection rate than the NAAT (i.e., RT-qPCR, Xpert and FilmArray).
In particular, the Lumipulse test judged an inconclusive result in a low viral load sample. In this case, the NATT test must be used to determine whether the sample is positive or negative. There was a high correlation between the Xpert Ct value and the RT-qPCR Ct value, viral load and antigen level. Of 165 samples, only one sample $(\mathrm{Ct}=38)$ was discordant between Xpert and RT-qPCR, which was attributed to a very low viral load. Collectively, Xpert yielded accurate test results and quantitatively evaluated the viral load in samples [23]. Therefore, Xpert is considered to be a particularly useful method for random access SARS-CoV-2 testing. 
(A)

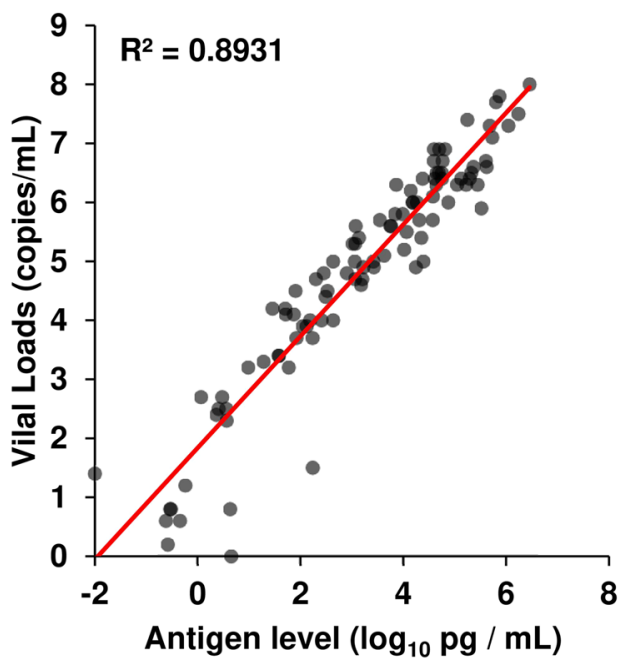

(C)

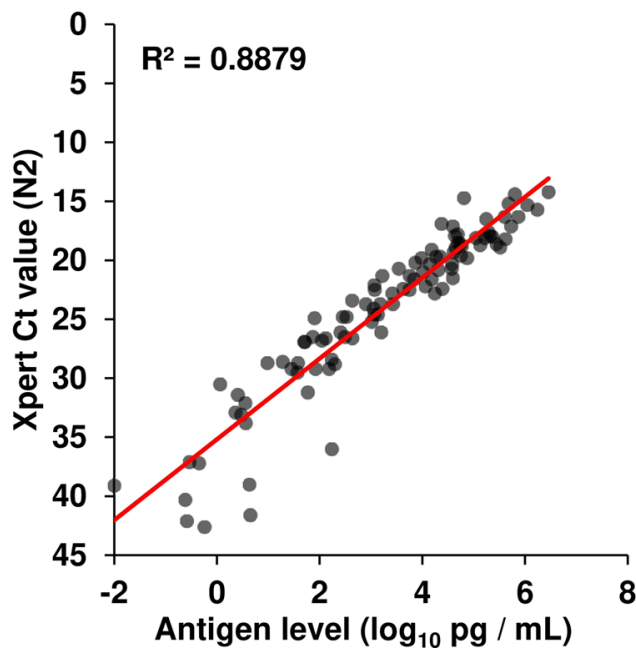

(B)

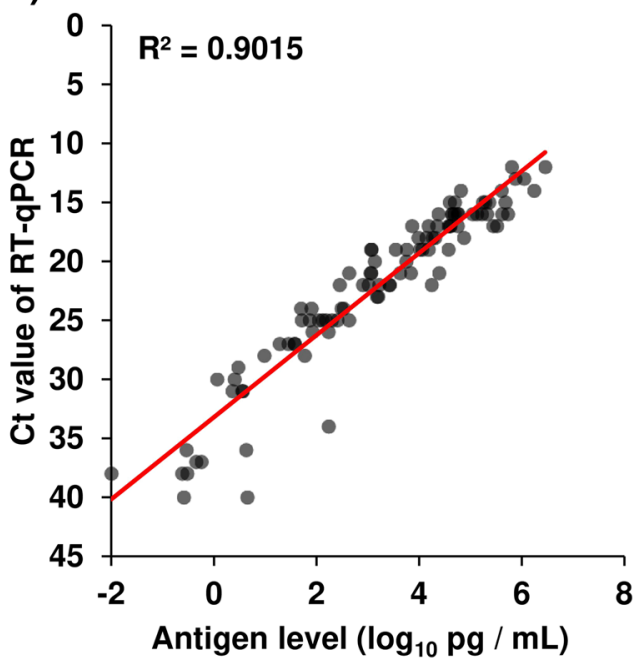

(D)

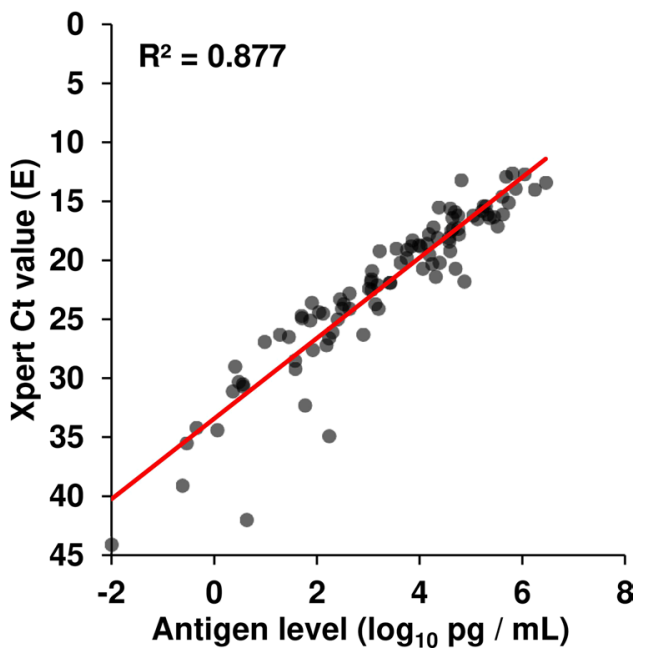

Fig. 4 Correlation between the antigen level, viral load and Ct value. A Correlation between the antigen level and the viral load determined by RT-qPCR. B Correlation between the antigen level and the Ct value of RT-qPCR. C, D Correlation between the antigen level and the Ct value of Xpert. Xpert was plotted for the Ct values of N2 (C) and E (D). The decision coefficient $\left(R^{2}\right)$ is shown in the scatter plot

Unlike the rapid antigen test (i.e., paper-based assays or lateral flow immunochromatography), the Lumipulse antigen test is based on the chemiluminescence enzyme immunoassay [10]. Compared with the assay performance of the rapid antigen test by other groups [24-29], our data show that the Lumipulse antigen test is relatively consistent with the NAAT analysis results among antigen tests $[12,30]$. In addition, the Lumipulse antigen test specifically determined a positive result with a high accuracy in specimens with a viral load of more than 100 copies/mL [10]. Our previous analysis showed that NAAT detected the virus for a long time after the onset of symptom, while the Lumipulse antigen test becomes negative [11]. It was often observed that Lumipulse antigen tests turned to be negative after 10 days of symptom onset [12], which is consistent with the time when infectious viruses are detected in vitro [31-33].

The use of random access automated testing is removing barriers to conventional PCR test. The PCR test is the gold standard, but its process is time consuming because a certain number of samples are collected and analyzed in batches. In addition, it requires a skill, which limits its use by personnel who are not familiar with the PCR test. In contrast, Xpert, FilmArray, and Lumipulse take advantage of random access detection, which is useful for emergency. The turnaround time for Xpert, 
FilmArray, and Lumipulse is within $45 \mathrm{~min}, 45 \mathrm{~min}$, and 35 min. In Xpert, if the amplification plot reaches the threshold, the test results can be returned in a shorter time. In addition, the simplicity of operation allows for testing to be performed in the absence of experienced personnel. Combining multiple testing methods will improve the speed and efficiency of the testing system in hospitals.

Using different tests that take advantage of on-demand nucleic acid amplification is an effective strategy. Xpert targets a small number of genes (often a single pathogen) due to the limited number of fluorescence wavelengths that can be detected and is inexpensive. In comparison, FilmArray amplifies several targets by multiplex PCR and then performs nested-PCR in multiple wells, which allows for a large number of target genes to be detected simultaneously, but it is more expensive. The $\mathrm{Ct}$ value and amplification curve can be confirmed using the Xpert system but not the FilmArray because it is a qualitative test. Therefore, the Xpert may be more advantageous for universal use in testing for SARS-CoV-2 in suspected patients, and the FilmArray may be more useful in patients with underlying medical conditions or children who are at risk of severe illness due to viral infections other than SARS-CoV-2.

In this study, we compared the Xpert Ct values with the RT-qPCR Ct values as a reference and found an extremely high correlation (Fig. 3A and B). The correlation was higher for Xpert (N2) than for Xpert (E), likely because RT-qPCR targets the $N$ gene. However, the absolute $\mathrm{Ct}$ values of RT-qPCR were closer to those of Xpert (E) than to those of Xpert (N2), except for samples with low viral loads (Tables 1 and 2). In some samples with low viral loads, the Xpert Ct (N2), but not the Xpert Ct (E), could be measured as previously reported [23].

The $\mathrm{Ct}$ value is useful as a surrogate indicator of viral load by RT-PCR. However, it should be carefully compared with other datasets because the $\mathrm{Ct}$ value changes depending on the following factors. First, it is depended on the target region of PCR amplification. In particular, in the case of in-house assays, it is necessary to carefully consider whether the same conditions are used for primer sequences, reagents, and measuring equipment. Next, the Ct value varies depending on the threshold setting. It is necessary to make sure that the threshold of the assay used is always at a certain level. Therefore, if the amount of virus is to be examined accurately, it is better to measure a standard sample and measure viral load quantitatively from the calibration curve.

Overall, the Xpert, FilmArray, and Lumipulse tests could detect SARS-CoV-2 in samples with sufficient viral loads. The only inconsistency was observed in specimens with low viral loads, but these patients likely release less live virus [31, 32, 34]. In fact, we previously demonstrated that discrepant results between the Lumipulse antigen test and RT-qPCR were attributable to low viral loads collected from seropositive patients [12]. Therefore, the use of random access detection may be effective for patient triage by enabling the rapid return of test results.

\section{Conclusions}

In this study, we evaluated the performance of two NAATs (Xpert and FilmArray) and a quantitative antigen test (Lumipulse) to detect SARS-CoV-2. When the result of RT-qPCR was used as reference, the test results of Xpert and FilmArray showed high agreement, indicating that these tests have almost equivalent test accuracy. The Lumipulse antigen test had a slightly lower concordance rate than Xpert and FilmArray, but had high test accuracy except for samples with low viral load. Xpert $\mathrm{Ct}$ value correlated with RT-qPCR Ct value, viral load and antigen level, helping to understand the phases of infection. Xpert, FilmArray and Lumipulse tests can be performed automatically on dedicated equipment, achieving seamless and accurate testing for SARS-CoV-2 without the need for complicated procedures.

\section{Acknowledgements}

We thank Hisahiro Tajima and Doura Teruya for technical discussions, all medical and ancillary hospital staff, and the patients for consenting to participate. We thank Melissa Crawford, PhD, from Edanz (https://jp.edanz.com/ac) for editing a draft of this manuscript.

\section{Authors' contributions}

$\mathrm{YH}$ : conceptualization, formal analysis, investigation, data visualization, statistical analysis and writing the original draft. MM, MS, YN1, YN2, KH, HS and KA: formal analysis, data collection and investigation. $\mathrm{MH}$ and $\mathrm{HM}$ : interpretation of data, supervision and project administration. TT and YK: acquisition of data, interpretation of data and sample collection. YM: acquisition of data, interpretation of data, sample collection and project administration. MO: conceptualization, supervision, project administration and review and edit the manuscript. All authors read and approved the final manuscript. YN1 and YN2 correspond to Yume Natori and Yuki Nagakubo, respectively.

\section{Funding}

This study was supported by a Grant-in-Aid for the Genome Research Project from Yamanashi Prefecture (to M.O. and Y.H.), the Japan Society for the Promotion of Science (JSPS) KAKENHI Early-Career Scientists JP18K16292 (to Y.H.), a Grant-in-Aid for Scientific Research (B) $20 \mathrm{H} 03668$ (to Y.H.), a Research Grant for Young Scholars (to Y.H.), the YASUDA Medical Foundation (to Y.H.), the Uehara Memorial Foundation (to Y.H.), and Medical Research Grants from the Takeda Science Foundation (to Y.H.). The funder had no role in the design of the study and collection, analysis, and interpretation of data and in writing the manuscript.

\section{Availability of data and materials}

The datasets used and/or analysed during the current study are available from the corresponding author on reasonable request.

\section{Declarations}

Ethics approval and consent to participate

The Institutional Review Board at Yamanashi Central Hospital approved this study and the use of an opt-out consent method (G-2019-1). The requirement 
for written informed consent was waived because this was an observational study. Patient participation in the study was optional.

\section{Consent for publication}

Not applicable.

\section{Competing interests}

The authors have no conflicts of interest.

\begin{abstract}
Author details
${ }^{1}$ Genome Analysis Center, Yamanashi Central Hospital, 1-1-1 Fujimi, Kofu, Yamanashi, Japan. ${ }^{2}$ Division of Microbiology in Clinical Laboratory, Yamanashi Central Hospital, 1-1-1 Fujimi, Kofu, Yamanashi, Japan. ${ }^{3}$ Division of Genetics and Clinical Laboratory, Yamanashi Central Hospital, 1-1-1 Fujimi, Kofu, Yamanashi, Japan. ${ }^{4}$ Central Clinical Laboratory, Yamanashi Central Hospital, 1-1-1 Fujimi, Kofu, Yamanashi, Japan. ${ }^{5}$ Department of Gastroenterology, Yamanashi Central Hospital, 1-1-1 Fujimi, Kofu, Yamanashi, Japan. ${ }^{6}$ Lung Cancer and Respiratory Disease Center, Yamanashi Central Hospital, 1-1-1 Fujimi, Kofu, Yamanashi, Japan. ${ }^{7}$ The University of Tokyo, 7-3-1 Hongo, Bunkyo-ku, Tokyo, Japan.
\end{abstract}

Received: 27 October 2021 Accepted: 19 February 2022

Published online: 04 March 2022

\section{References}

1. World Health Organization: Tracking SARS-CoV-2 variants. https://www. whoint/en/activities/tracking-SARS-CoV-2-variants/.

2. Centers for Disease Control and Prevention (CDC): Emerging SARS-CoV-2 Variants. https://www.cdcgov/coronavirus/2019-ncov/more/scienceand-research/scientific-brief-emerging-variantshtml 2021.

3. European Centre for Disease Prevention and Control: SARS-CoV-2 variants of concern. https://www.ecdceuropaeu/en/covid-19/variants-concern.

4. Hirotsu Y, Omata M. Discovery of a SARS-CoV-2 variant from the P.1 lineage harboring K417T/E484K/N501Y mutations in Kofu, Japan. J Infect. 2021;82(6):276-316.

5. Hirotsu Y, Omata M. Detection of R.1 lineage severe acute respiratory syndrome coronavirus 2 (SARS-CoV-2) with spike protein W152L/E484K G769V mutations in Japan. PLoS Pathog. 2021;17(6):e1009619.

6. Hadfield J, Megill C, Bell SM, Huddleston J, Potter B, Callender C, Sagulenko P, Bedford T, Neher RA. Nextstrain: real-time tracking of pathogen evolution. Bioinformatics. 2018;34(23):4121-3.

7. Hirotsu Y, Omata M. SARS-CoV-2 B.1.1.7 lineage rapidly spreads and replaces R. 1 lineage in Japan: serial and stationary observation in a community. Infect, Genet Evol. 2021;95:105088.

8. Hirotsu Y, Omata M. Discovery of SARS-CoV-2 strain of P.1 lineage harboring K417T/ E484K / N501Y by whole genome sequencing in the city, Japan. medRxiv 2021:2021.2002.2024.21251892.

9. Hirotsu Y, Mochizuki H, Omata M. Double-quencher probes improve detection sensitivity toward Severe Acute Respiratory Syndrome Coronavirus 2 (SARS-CoV-2) in a reverse-transcription polymerase chain reaction (RT-PCR) assay. J Virol Methods. 2020;284:113926.

10. Hirotsu Y, Maejima M, Shibusawa M, Nagakubo Y, Hosaka K, Amemiya K, Sueki H, Hayakawa M, Mochizuki H, Tsutsui T et al. Comparison of automated SARS-CoV-2 antigen test for COVID-19 infection with quantitative RT-PCR using 313 nasopharyngeal swabs including from 7 serially followed patients. Int J Infect Dis. 2020

11. Hirotsu Y, Maejima M, Shibusawa M, Amemiya K, Nagakubo Y, Hosaka K, Sueki H, Hayakawa M, Mochizuki H, Tsutsui T, et al. Analysis of a persistent viral shedding patient infected with SARS-CoV-2 by RT-qPCR, FilmArray Respiratory Panel v2.1, and antigen detection. J Infect Chemother. 2020;27:406.

12. Hirotsu Y, Maejima M, Shibusawa M, Amemiya K, Nagakubo Y, Hosaka K, Sueki H, Hayakawa M, Mochizuki H, Tsutsui T, et al. Prospective study of 1,308 nasopharyngeal swabs from 1,033 patients using the LUMIPULSE SARS-CoV-2 antigen test: comparison with RT-qPCR. Int J Infect Dis. 2021;105:7.

13. Broder K, Babiker A, Myers C, White $T$, Jones $H$, Cardella J, Burd EM, Hill CE, Kraft CS, McAdam AJ. Test agreement between Roche Cobas 6800 and Cepheid GeneXpert Xpress SARS-CoV-2 assays at high cycle threshold ranges. J Clin Microbiol. 2020;58(8):e01187-e11120.
14. Goldenberger D, Leuzinger K, Sogaard KK, Gosert R, Roloff T, Naegele K, Cuenod A, Mari A, Seth-Smith H, Rentsch K, et al. Brief validation of the novel GeneXpert Xpress SARS-CoV-2 PCR assay. J Virol Methods. 2020;284:113925

15. Moran A, Beavis KG, Matushek SM, Ciaglia C, Francois N, Tesic V, Love $\mathrm{N}$, McAdam AJ. Detection of SARS-CoV-2 by use of the Cepheid Xpert Xpress SARS-CoV-2 and Roche Cobas SARS-CoV-2 assays. J Clin Microbiol. 2020;58(8):e00772-e1720.

16. Wolters F, van de Bovenkamp J, van den Bosch B, van den Brink S, Broeders M, Chung NH, Favié B, Goderski G, Kuijpers J, Overdevest I, et al. Multicenter evaluation of cepheid xpert ${ }^{\circledR}$ xpress SARS-CoV-2 point-of-care test during the SARS-CoV-2 pandemic. J Clin Virol. 2020;128:104426.

17. Lieberman JA, Pepper G, Naccache SN, Huang M-L, Jerome KR, Greninger AL, McAdam AJ. Comparison of commercially available and laboratorydeveloped assays for $<\mathrm{i}>\ln$ Vitro $</ \mathrm{i}>$ detection of SARS-CoV-2 in clinical laboratories. J Clin Microbiol. 2020;58(8):e00821-e1820.

18. Hirotsu Y, Maejima M, Nakajima M, Mochizuki H, Omata M. Environmental cleaning is effective for the eradication of severe acute respiratory syndrome coronavirus 2 (SARS-CoV-2) in contaminated hospital rooms: a patient from the Diamond Princess cruise ship. Infect Control Hosp Epidemiol. 2020;41(9):1105-6.

19. Hirotsu Y, Maejima M, Shibusawa M, Nagakubo Y, Hosaka K, Amemiya K, Sueki H, Hayakawa M, Mochizuki H, Omata M. Pooling RT-PCR test of SARS-CoV-2 for large cohort of 'healthy' and infection-suspected patients: a prospective and consecutive study on 1,000 individuals. medRxiv. 2020;38:905.

20. Shirato K, Nao N, Katano H, Takayama I, Saito S, Kato F, Katoh H, Sakata M, Nakatsu Y, Mori Y, et al. Development of genetic diagnostic methods for novel coronavirus 2019 (nCoV-2019) in Japan. Jpn J Infect Dis. 2020;73(4):304-7.

21. Hirotsu Y, Maejima M, Shibusawa M, Amemiya K, Nagakubo Y, Hosaka K, Sueki H, Mochizuki H, Tsutsui T, Kakizaki Y, et al. Analysis of Covid-19 and non-Covid-19 viruses, including influenza viruses, to determine the influence of intensive preventive measures in Japan. J Clin Virol. 2020;129:104543.

22. Landis JR, Koch GG. The measurement of observer agreement for categorical data. Biometrics. 1977;33(1):159-74.

23. Loeffelholz MJ, Alland D, Butler-Wu SM, Pandey U, Perno CF, Nava A, Carroll KC, Mostafa H, Davies E, McEwan A, et al. Multicenter evaluation of the cepheid Xpert Xpress SARS-CoV-2 test. J Clin Microbiol. 2020;58(8):e00926-e1920.

24. Kuo P, Realegeno S, Pride DT. Comparison of two nucleic acid amplification tests (NAATs) and two antigen tests for detection of SARS-CoV-2 from upper respiratory specimens. J Clin Virol Plus. 2021;1(1-2):100011.

25. Sood N, Shetgiri R, Rodriguez A, Jimenez D, Treminino S, Daflos A, Simon P. Evaluation of the Abbott BinaxNOW rapid antigen test for SARS-CoV-2 infection in children: implications for screening in a school setting. PLoS ONE. 2021;16(4):e0249710.

26. Scohy A, Anantharajah A, Bodeus M, Kabamba-Mukadi B, Verroken A, Rodriguez-Villalobos H. Low performance of rapid antigen detection test as frontline testing for COVID-19 diagnosis. J Clin Virol. 2020;129:104455.

27. Mak GC, Cheng PK, Lau SS, Wong KK, Lau CS, Lam ET, Chan RC, Tsang DN. Evaluation of rapid antigen test for detection of SARS-CoV-2 virus. J Clin Virol. 2020;129:104500.

28. Cerutti F, Burdino E, Milia MG, Allice T, Gregori G, Bruzzone B, Ghisetti V. Urgent need of rapid tests for SARS CoV-2 antigen detection: evaluation of the SD-Biosensor antigen test for SARS-CoV-2. J Clin Virol. 2020;132:104654.

29. Albert E, Torres I, Bueno F, Huntley D, Molla E, Fernández-Fuentes MÁ, Martínez M, Poujois S, Forqué L, Valdivia A, et al. Field evaluation of a rapid antigen test (Panbio COVID-19 Ag Rapid Test Device) for COVID-19 diagnosis in primary healthcare centers. Clin Microbiol Infect. 2020;58:e00977.

30. Hirotsu Y, Sugiura H, Maejima M, Hayakawa M, Mochizuki H, Tsutsui T, Kakizaki Y, Miyashita Y, Omata M. Comparison of Roche and Lumipulse quantitative SARS-CoV-2 antigen test performance using automated systems for the diagnosis of COVID-19. Int J Infect Dis. 2021;108:263-9.

31. Wolfel R, Corman VM, Guggemos W, Seilmaier M, Zange S, Muller MA, Niemeyer D, Jones TC, Vollmar P, Rothe C et al. Virological assessment of hospitalized patients with COVID-2019. Nature. 2020. 
32. Bullard J, Dust K, Funk D, Strong JE, Alexander D, Garnett L, Boodman C, Bello A, Hedley A, Schiffman Z et al. Predicting infectious SARS-CoV-2 from diagnostic samples. Clin Infect Dis. 2020.

33. Perera R, Tso E, Tsang OTY, Tsang DNC, Fung K, Leung YWY, Chin AWH, Chu DKW, Cheng SMS, Poon LLM, et al. SARS-CoV-2 virus culture and subgenomic RNA for respiratory specimens from patients with mild coronavirus disease. Emerg Infect Dis. 2020;26(11):2701-4.

34. Munster VJ, Feldmann F, Williamson BN, van Doremalen N, Pérez-Pérez L, Schulz J, Meade-White K, Okumura A, Callison J, Brumbaugh B, et al. Respiratory disease in rhesus macaques inoculated with SARS-CoV-2. Nature. 2020;585(7824):268-72.

\section{Publisher's Note}

Springer Nature remains neutral with regard to jurisdictional claims in published maps and institutional affiliations.

- fast, convenient online submission

- thorough peer review by experienced researchers in your field

- rapid publication on acceptance

- support for research data, including large and complex data types

- gold Open Access which fosters wider collaboration and increased citations

- maximum visibility for your research: over $100 \mathrm{M}$ website views per year

At BMC, research is always in progress.

Learn more biomedcentral.com/submissions 